\title{
Chronic Bilateral Tibial Stress Fractures with Valgus Treated with Bilateral Intramedullary Nailing: A Case Report
}

\author{
Steven K Dailey ${ }^{1}$, Michael T. Archdeacon ${ }^{2}$
}

What to Learn from this Article?

A rare presentation of bilateral tibia stress fracture in young female: diagnostic and management challenges

Abstract

Introduction: Stress fractures are overuse injuries most commonly seen in athletes, military recruits, and individuals with endocrine abnormalities. It has been demonstrated that chronic cases of anterior tibial stress fractures refractory to conservative management respond well to intramedullary nailing. To our knowledge, only one report has been published concerning patients with bilateral tibial stress fractures treated with bilateral intramedullary nailing. All patients in the series were high-level athletes. We present the case of a non-athletic patient with chronic bilateral tibial stress fractures and associated deformity successfully treated with bilateral intramedullary nails.

Case Report: A 23-year-old Caucasian female full-time student presented with chronic bilateral shin pain for approximately five years. She had failed an extensive regimen of conservative management. She was diagnosed with chronic bilateral tibial stress fractures based on history, physical examination, and radiologic findings. She subsequently underwent sequential intramedullary nailing of her tibiae. Both tibiae were in valgus alignment; however, this did not preclude nail placement. The nails deformed upon insertion into the sclerotic canals to conform to the deformation. Post operatively the tibiae united and patient was relieved of her symptoms.

Conclusion: Bilateral intramedullary nailing of chronic bilateral tibial stress fractures should be considered as a treatment option for all patients, not just high-level athletes, who fail a trial of conservative management. Additionally, mild to moderate tibial malalignment does not necessarily preclude tibial nailing as the smaller nails placed in sclerotic canals will likely deform on insertion and conform to the canal.

Keywords: Bilateral intramedullary nailing, bilateral tibial stress fractures, valgus.

Author's Photo Gallery

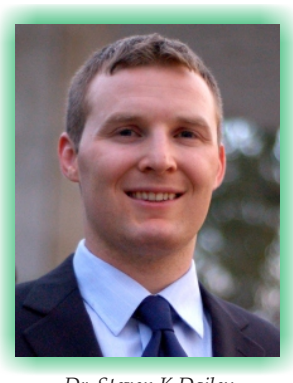

Dr. Steven K Dailey

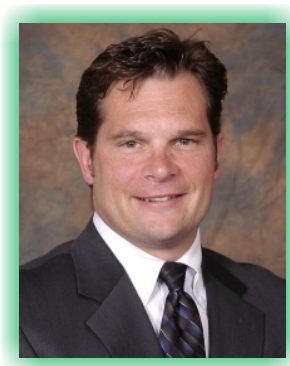

Dr. Michael T Archdeacon

${ }^{1}$ Department of Orthopaedic Surgery, University of Cincinnati, PO Box 670212, Cincinnati, OH 45267-0212

\section{Address of Correspondence}

Dr. Steven Dailey,

University of Cincinnati, Dept of Orthopaedic Surgery, 231 Albert Sabin Way, ML 212 Cincinnati, OH 45267-0212.

Email: steven.dailey@uc.edu

$t$ (513) 558-1109 / f (513) 558-2220

\section{Introduction}

Stress fractures are overuse injuries most commonly seen in athletes, military recruits, and individuals with endocrine abnormalities [1-5]. They most frequently involve the weight bearing bones of the lower extremity with the tibia comprising between $18.9 \%$ to $63.0 \%$ of reported cases [5-7]. A subset of tibial stress fractures are located in the anterior cortex. Tensile forces coupled with poor vascularity predispose this area to an increased risk of delayed union, nonunion, or complete fracture [3]. It has been demonstrated in the literature that chronic cases of anterior tibial stress fractures refractory to conservative management respond well to intramedullary nailing $[1,2,6,8]$. To our knowledge, only one report has been published concerning patients with bilateral tibial stress fractures treated with bilateral intramedullary nailing [6]. All patients in the series were high-level athletes [6]. We present the case of a non-athletic patient with chronic bilateral tibial stress fractures and associated deformity successfully treated with bilateral intramedullary nails. 



Figure 1: Anteroposterior (Fig. 1-a) and lateral (Fig. 1-b) radiographs of the left tibia demonstrate valgus bowing, cortical periosteal reaction, and a narrow medullary canal.

\section{Case Report}

A 23-year-old Caucasian female full-time student presented with chronic bilateral shin pain for approximately five years. She had failed an extensive regimen of conservative management with orthotic insoles, NSAIDs, vitamin D, physical therapy, and corticosteroid injections. She also underwent bilateral compartment release at an outside institution for suspected exertional compartment syndrome which did not resolve her symptoms. After seeing pain management and failing a spinal cord stimulator trial, she was referred to us for evaluation of her tibiae.

The patient described a constant, burning, $8-10 / 10$ pain from the knee to mid tibia. Her pain was isolated to the anterior leg and was exacerbated by rising from a seated position, prolonged standing, and walking. She denied back, hip, knee or ankle pain. Her sleep and activities of daily living had been limited secondary to pain. Her standing tolerance was 10 minutes and walking tolerance

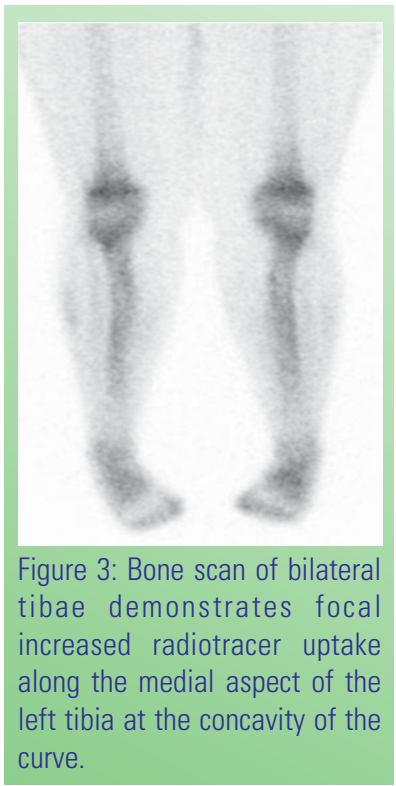

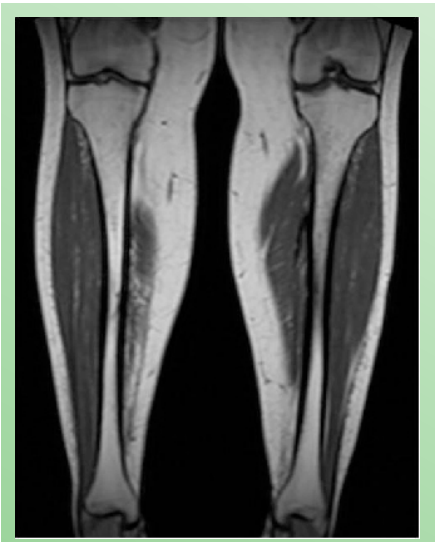

Figure 4: Coronal section of MRI without contrast demonstrated hyper-intense fluid, edema, and cortical thickening along the anteromedial border of the mid tibiae bilaterally.



Figure 2: Anteroposterior (Fig. 2-a) and lateral (Fig. 2-b) radiographs of the right tibia demonstrate valgus bowing, cortical periosteal reaction, and a narrow medullary canal.

was one quarter mile.

Inspection of the patient's lower extremities revealed a valgus malalignment of her tibiae. Her active and passive range of motion was full in her lumbar spine, hips, knees, and ankles bilaterally. Well-healed incisions along the tibial crest were present which extended $10 \mathrm{~cm}$ in a parasagittal fashion. There was mild bruising over the tibial crest which was slightly warm and significantly tender to palpation. Resisted knee flexion and plantar flexion was moderately painful bilaterally in the mid tibia. Manual muscle testing was $5 / 5$ through proximal leg muscles and 5-/5 in bilateral ankle dorsiflexion. Her gait showed mildly shortened stride length, but was smooth, symmetric, and without an obvious antalgic component.

Pre-operative plain radiographs [Figs. 1A-B and 2A-B] of her bilateral tibiae demonstrated thickening of the cortices with narrowing of the medullary canal which was more prominent on the left. AP films revealed valgus bowing of the tibiae measured at $8.5^{\circ}$ and $6^{\circ}$ on the left and right respectively. Lateral views showed recurvatum of $3^{\circ}$ on the left and $5^{\circ}$ on the right. A bone scan demonstrated mild focal increased uptake along the medial aspect of the left tibia [Fig. 3]. MRI of her lower extremities demonstrated postsurgical changes in the mid aspect of the lower legs with hyperintense fluid, edema, and cortical thickening along the anteromedial border of the mid tibiae bilaterally [Fig. 4]. Based on history, physical exam, and radiographic changes, the patient was diagnosed with chronic bilateral tibial stress fractures.

Due to failure of extensive treatment including nonoperative and operative management, the patient decided to proceed with intramedullary nail stabilization of her left tibia. Intra-operatively, the intramedullary canal was noted to be extensively sclerotic requiring an initial reaming with a $6.5 \mathrm{~mm}$ reamer, advancing in $0.5 \mathrm{~mm}$ increments up to 11 


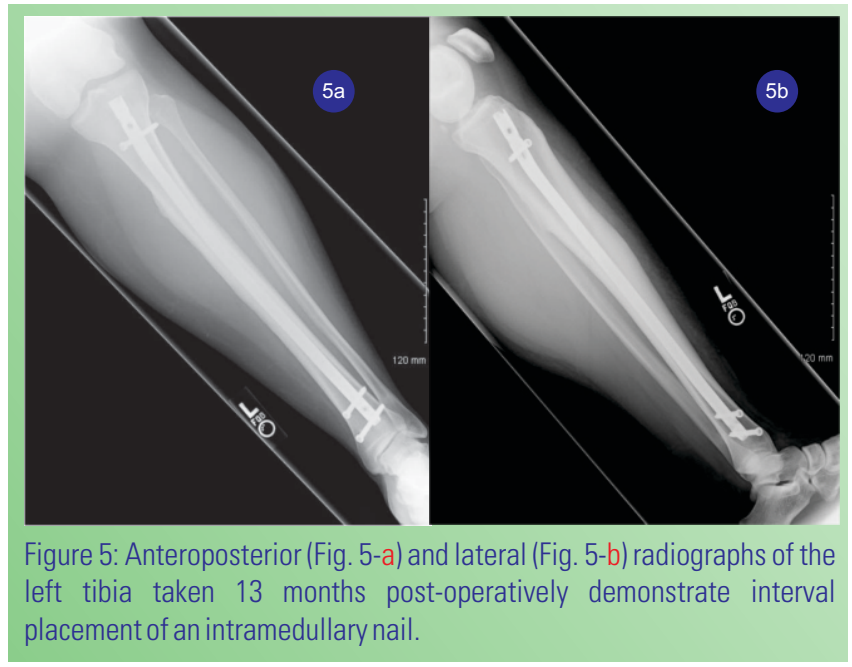

$\mathrm{mm}$. Of significant note is the observed deformation of the intramedullary nail after placement. Her left tibia had a valgus alignment of $6.0^{\circ}$; however, as the nail was placed within the sclerotic intramedullary canal, it deformed during insertion to conform to the valgus alignment of the tibial shaft. The nail was locked proximally with a single dynamic locking screw and distally with two static screws.

The patient was made weight bearing as tolerated and prescribed outpatient physical therapy. At 3 months postop, she reported to clinic with complete resolution of symptoms in her left tibia. Due to consistent unresolved pain on her contralateral side, she elected to undergo operative fixation of her right tibia, and intramedullary nailing was performed in a similar manner. Again, as the IMN was placed within the sclerotic intramedullary canal, the nail deformed to comply with the $8.5^{\circ}$ valgus alignment of the right tibia

At six and nine months status post IMN stabilization of her right and left tibial stress fractures, the patient was ambulating without assistance and had minimal complaints other than mild intermittent pain with jogging. Gait demonstrated normal station and no evidence of an antalgic gait. There was minimal to no tenderness of the knees or tibiae bilaterally with palpation. Knee range of motion was 0 to $130^{\circ}$ bilaterally and without instability. She was neurovascularly intact with fully healed skin incisions. Radiographs, including AP and lateral of the right and left tibiae, demonstrated no evidence of fracture and intact hardware [Figs. 5A-B and 6A-B]. The patient responded very well to bilateral intramedullary nailing and was advised to continue her activities as tolerated. Of note, the mild to moderate tibial malalignment did not preclude tibial nailing as the smaller nails placed in sclerotic canals deformed on insertion to comply with the valgus alignment of the tibia.

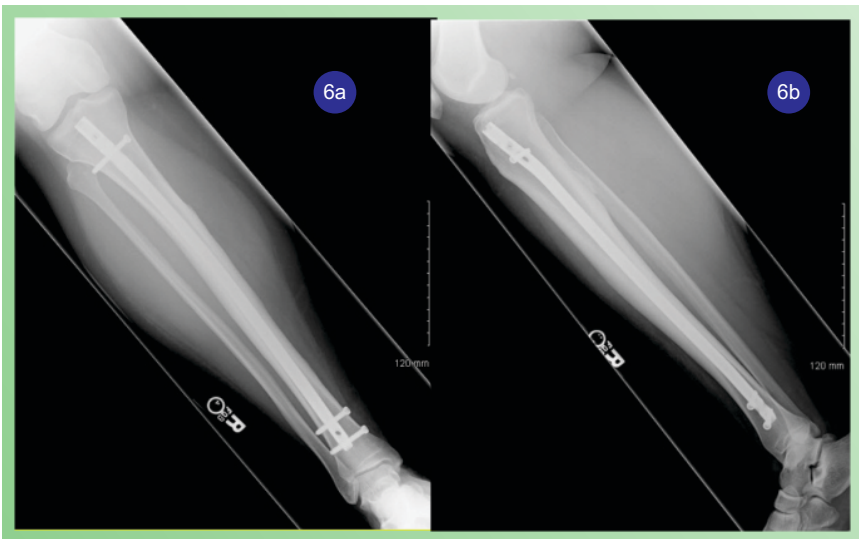

Figure 6: Anteroposterior (Fig. 6-a) and lateral (Fig. 6-b) radiographs of the right tibia taken 13 months post-operatively demonstrate interval placement of an intramedullary nail.

\section{Discussion}

Stress fractures were first described in 1855 by Briethaupt [9]. They are especially prevalent in athletes, military recruits, and patients with endocrine or nutritional abnormalities [1-4, 10]. Stress fractures result from dynamic cyclic loading $[1,5$, $10,13]$. Excessive, repetitive, submaximal loads result in an imbalance between bone formation and resorption. Osteoclastic activity, as a consequence of loading, occurs faster than osteoblastic activity and can result in bony resorption [1]. This dynamic is particularly concerning for anterior midtibial stress fractures. The tension side of the tibia has less blood supply and musculotendinous support when compared to the posteromedial cortex. When repetitive forces are applied to this poorly vascularized area, the bone may not be able to heal before a new insult occurs $[3,5]$. This predisposes the fracture to being refractory to conservative management, and it may progress to complete fracture, nonunion, or focal bone infarct $[2,3,6]$.

Patients often present with an insidious onset of pain over a 23 week period in the mid-shaft of their tibia $[3,5,6]$. The pain is worse with activities and relieved by rest $[5,6]$. They may describe a recent increase in athletic activities or change in shoes or equipment [3,6]. Physical exam typically reveals point tenderness over the affected bone, local swelling, palpable periosteal thickening, or functional testing that reproduces pain [3,5]. Evaluation of biomechanics may demonstrate a muscle imbalance or limb length discrepancy [3].

Plain radiographs are the initial imaging modality of choice; however, radiographic changes typically lag behind clinical symptoms by 2 weeks to 3 months and may never be evident $[3,5,6,10,14]$. In fact, up to $85 \%$ of stress fractures are overlooked on first, and $50 \%$ on second radiograph [10]. Initial changes include a subtle radiolucency or poor 
definition of the cortex. Later findings demonstrate and 3 months radiographically. All patients reported thickening and sclerosis of the endosteum and periosteal satisfaction with the procedure and were able to pursue new bone formation. The anterior cortex of the tibia may be athletic endeavors. In our case, the patient was not a high level thickened and show a lucent line, the "dreaded black line [3, athlete and had a five year course before definitive treatment.

$5,6,8]$." If a stress fracture is suspected, and initial films are non-diagnostic, repeat films obtained 2 weeks after initial imaging may reveal a fracture [3].

\section{Conclusion}

Technetium-99m-labeled diphosphonate bone scintigraphy is an excellent option should plain film prove equivocal. It is typically abnormal within 6-72 hours of symptom onset [10]. Sensitivity has been described to approach $100 \%$; however, specificity is not as high $[5,11$, 14]. Reports suggest that $20-40 \%$ of lesions seen on bone scintigraphy were asymptomatic [10]. Acute stress fractures are depicted as discrete, localized, sometimes linear areas of increased uptake on all 3 phases of the scan [5]. MRI, CT, and ultrasound have been used to effectively diagnose stress fractures, but are typically reserved for cases in which plain films and bone scintigraphy yield equivocal results.

The majority of tibial stress fractures will respond to rest, activity modification and correction of biomechanical abnormalities; furthermore, intrinsic factors such as hormonal or nutritional abnormalities should be assessed and corrected $[1-4,10]$. Alternatively, stress fractures of the mid-anterior tibia have been described as a specific subset of tibial stress fractures that have mixed results with nonoperative therapy $[1,2,4,11,12]$. Notably, Beals and Cook described a series of 20 patients of which only eight were able to return to sport with rest alone [11]. Additionally, five of eight patients that were allowed full activity developed a complete fracture. These Case series demonstrate that anterior mid tibial stress fractures have an increased predilection for progressing to complete fracture, delayed union, or non-union. Thus, after a trial of non-operative therapy, they often require more aggressive management. The use of intramedullary tibial nails has been described $[1,2,6,8]$.

Varner et al. have been the only authors to publish on the treatment of bilateral tibial stress fractures with bilateral tibial nailing [6]. They describe successfully treating four patients with bilateral tibial stress fractures. Their cohort included 7 collegiate athletes with 11 chronic anterior midtibial stress fractures who were treated with reamed intramedullary nailing. Symptom duration averaged of 12 months, and patients were treated non-operatively for a minimum of four months. Post-operatively, stress fractures in this cohort were found to unite at 2.7 months clinically

Bilateral intramedullary nailing of chronic bilateral tibial stress fractures should be considered as a treatment option for all patients, not just high-level athletes, who fail a trial of conservative management. Additionally, mild to moderate tibial malalignment does not necessarily preclude tibial nailing as the smaller nails placed in sclerotic canals will likely deform on insertion and conform to the canal.

\section{Clinical Message}

Bilateral tibial stress fractures that are recalcitrant to conservative management may benefit from fixation with bilateral intramedullary nails. These intramedullary nails can be placed even in the presence of mild to moderate tibial malalignment with good clinical results.

\section{References}

1. Barrick EF, Jackson CB. Prophylactic intramedullary fixation of the tibia for stress fracture in a professional athlete. J Orthop Trauma. 1992;6(2):241-4.

2. Chang PS, Harris RM. Intramedullary nailing for chronic tibial stress fractures. A review offive cases. Am J Sports Med. 1996;24(5):688-92.

3. Shindle MK, Endo Y, Warren RF, et al. Stress fractures about the tibia, foot, and ankle. J Am Acad Orthop Surg. 2012;20(3):167-76.

4. Batt ME, Kemp S, Kerslake R: Delayed union stress fractures of the anterior tibia: Conservative management. Br JSports Med 2001;35(1):74-77.

5. Boden BP, Osbahr DC. High-risk stress fractures: evaluation and treatment. J Am Acad Orthop Surg. 2000;8(6):344-53.

6 Varner KE, Younas SA, Lintner DM, Marymont JV. Chronic anterior midtibial stress fractures in athletes treated with reamed intramedullary nailing. Am J Sports Med. 2005;33(7):1071-6.

7. Snyder RA, Koester MC, Dunn WR. Epidemiology of stress fractures. Clin Sports Med. 2006;25(1):37-52, viii.

8. Brukner P, Fanton G, Bergman AG, Beaulieu C, Matheson GO. Bilateral stress fractures of the anterior part of the tibial cortex. A case report. JBone Joint Surg Am. 2000;82(2):213-8.

9. Defoort S, Mertens P. Multiple tibial insufficiency fractures in the same tibia: a case report. Geriatr Orthop Surg Rehabil. 2011;2(2):69-72.

10. Moran DS, Evans RK, Hadad E. Imaging of lower extremity stress fracture injuries. Sports Med. 2008;38(4):345-56.

11. Beals RK, Cook RD: Stress fractures of the anterior tibial diaphysi\%

Journal of Orthopaedic Case Reports | Volume 4 | Issue 1 | Jan-Mar 2014 | Page 39-43 
Orthopedics 1991;14(8):869-875.

2013;55(2):359-66.

12. Rettig AC, Shelbourne KD, McCarroll JR, Bisesi M, Watts J: The 14. Sofka CM. Imaging of stress fractures. Clin Sports Med. natural history and treatment of delayed union stress fractures of the 2006;25(1):53-62, viii. anterior cortex of the tibia. Am J Sports Med 1988;16(3):250-255.

13. Cosman F, Ruffing J, Zion M, et al. Determinants of stress fracture risk in United States Military Academy cadets. Bone.

Conflict of Interest: Nil Source of Support: None
How to Cite this Article:

Dailey SK, Archdeacon MT. Chronic Bilateral Tibial Stress Fractures with Deformity Treated with Bilateral Intramedullary Nailing: A Case Report - Journal of Orthopaedic Case Reports. 2014 Jan-Mar;4(1):39-43 